\title{
Percepciones de estudiantes universitarios \\ hacia la corrección colaborativa de errores y el feedback virtual
}

\author{
University \\ Student's \\ Perceptions \\ towards \\ Collaborative \\ Correction of \\ Errors and Virtual \\ Feedback
}

\author{
Percepções \\ de estudantes \\ universitários \\ sobre a revisão \\ colaborativa de \\ erros e o feedback \\ virtual
}

Mabel Ortiz Navarrete** http://orcid.org/0000-0001-7481-1293

Claudio Díaz Larenas*** http://orcid.org/0000-0003-2394-2378

Claudine Benoit Ríos**** https://orcid.org/0000-0002-1791-2212

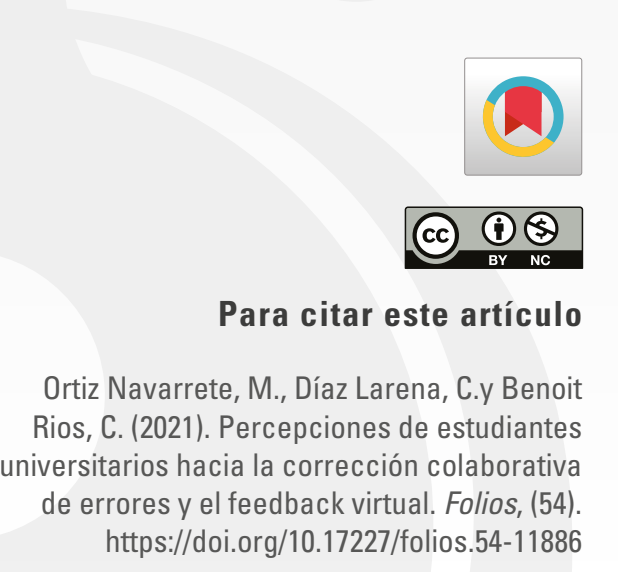

* El artículo se inserta en contexto del proyecto Fondecyt $\mathrm{N}^{0} 11150273$, denominado: Uso de estrategias de feedback correctivo escrito focalizado en un entorno virtual colaborativo Google Docs: Impacto y plan de capacitación.

** Doctora en Lingüística Universidad de Concepción, Chile. Profesora Universidad Católica de la Santísima Concepción, Chile.

Correo: mortiz@ucsc.cl

*** Doctor en Educación Universidad de Concepción, Chile. Profesora Universidad de Concepción, Chile.

Correo: claudiodiaz@udec.cl

**** Doctora en Lingüística Universidad de Concepción, Chile. Universidad Católica de la Santísima Concepción, Chile.

Correo: cbenoit@ucsc.cl 


\title{
Resumen
}

El objetivo de este artículo de investigación es dar a conocer las percepciones de 44 estudiantes de un programa de formación de profesores de inglés, acerca de la entrega de feedback correctivo y de la corrección colaborativa de errores durante la escritura colaborativa de un ensayo argumentativo en un entorno virtual Google Docs. Después de la aplicación de una entrevista semiestructurada y de la utilización de la técnica de análisis de contenido, se puede señalar que los estudiantes entrevistados consideran ventajas y algunas desventajas con respecto a la entrega de feedback a través de un entorno virtual Google Docs y a la corrección colaborativa de errores. Con respecto a las ventajas, se puede concluir que Google Docs es considerado un entorno que facilita la provisión de feedback. A su vez, la corrección colaborativa promueve la discusión y la disipación de dudas. Por otra parte, con respecto a las desventajas, se puede mencionar que no todos los estudiantes asumen de la misma manera la responsabilidad dentro de un grupo, y a raíz de esto, algunos estudiantes se inclinan por el trabajo individual.

\author{
Palabras clave \\ percepción; feedback correctivo; entorno virtual Google Docs; \\ trabajo colaborativo; corrección de errores
}

\begin{abstract}
The objective of this research article is to present the perceptions of 44 students of an English teacher training program, about the delivery of corrective feedback and the collaborative correction of errors during the writing of an argumentative essay in Google Docs. After the application of a semi-structured interview and the use of the content analysis technique, it can be noted that the interviewed students consider advantages and disadvantages with respect to the delivery of feedback through a virtual Google Docs environment and the collaborative correction of mistakes. Regarding the advantages, it can be concluded that Google Docs is considered an environment that facilitates the provision of feedback and promotes discussion and the dissipation of doubts. On the other hand, regarding the disadvantages, it can be mentioned that not all students assume responsibility within a group in the same way, and as a result, some students lean towards individual work.
\end{abstract}

\author{
Keywords \\ perception; focused corrective feedback; \\ Google Docs environment; collaborative work; error correction
}

\section{Resumo}

0 objetivo deste artigo de pesquisa é apresentar as percepções de 44 alunos de um programa de treinamento de professores de inglês sobre a entrega de feedback corretivo e da revisão colaborativa durante a escrita coletiva de um ensaio argumentativo em um ambiente virtual do Google Docs. Após a entrevista semiestruturada e a utilização da técnica de análise de conteúdo, pode-se apontar que os alunos entrevistados consideram vantagens e desvantagens a respeito do feedback no ambiente virtual Google Docs e à revisão colaborativa. Em relação às vantagens, pode-se concluir que o Google Docs é considerado um ambiente que facilita o fornecimento de feedback. Por sua vez, a revisão colaborativa promove a discussão e dissipação de dúvidas. Quanto às desvantagens, pode-se citar que nem todos os alunos assumem responsabilidades dentro de um grupo da mesma forma e, por isso, alguns alunos tendem para o trabalho individual.

\section{Palavras chave}

percepção; feedback corretivo focado; ambiente virtual do Google

Docs; trabalho colaborativo; correção de erros 


\section{Introducción}

En el marco del currículum chileno, se considera que el dominio de idiomas extranjeros es una exigencia en el actual contexto de relaciones globales. Por esta razón, el aprendizaje del inglés se ha convertido en una necesidad y un objetivo prioritario en la formación académica de un estudiante chileno, y aún más, cuando el foco de estudio se sitúa en un contexto de adquirir conocimiento de la lengua para enseñarla en un futuro. Desde esta perspectiva, la competencia en idioma inglés debe ser superior a alguien que adquiere la lengua para fines comunicativos. En la adquisición de una segunda lengua (L2), y principalmente dentro de un ambiente donde el inglés no se encuentra en un contexto inmediato, la producción escrita es una de las habilidades más difíciles de desarrollar (Kessler, 2009; Myles, 2002; Warschauer, 2010). Esta habilidad involucra procesos complejos, siendo una problemática recurrente los tipos de errores lingüísticos que cometen los estudiantes y cómo han de ser abordados. Esto es considerado una problemática en la escritura académica y más aún en un contexto universitario. Algunas investigaciones señalan que los docentes universitarios, en situaciones donde el idioma inglés no se encuentra en un contexto inmediato, consideran "los errores gramaticales como distractores y estigmatizadores, puesto que pueden interferir en la comprensión del mensaje" (Ferris y Roberts, 2001, p. 14). Por ende, la precisión gramatical es uno de los principales focos de atención de los profesores de inglés en Chile y, por esta razón, adquiere importancia en el presente estudio.

En el contexto del feedback, se puede señalar que, en la actualidad, las tecnologías junto con el uso de metodologías más activas en la enseñanza, como lo es la metodología colaborativa, permiten nuevas formas de abordar los errores gramaticales durante la producción de un texto. Desde este ámbito, en el presente artículo se describen las percepciones de estudiantes de un programa de formación de profesores de inglés en Chile.

En el proceso de adquisición de una L2, una de las inquietudes que surgen con frecuencia es cómo tratar los errores gramaticales que cometen los estudiantes. Si bien algunas estructuras pueden ser lingüísticamente fáciles, no son fáciles de internalizar por hablantes no nativos, lo que es reafirmado por investigaciones en las que se ha observado que no hay una relación directa entre la complejidad lingüística y el aprendizaje (Lightbown y Spada, 2008). Esto podría explicar por qué algunos estudiantes avanzan en su competencia comunicativa, pero no así en su competencia gramatical.

El tratamiento de los errores supone un gran reto para el profesor, puesto que las decisiones sobre "cómo y cuándo se deberían corregir los errores y quién debería hacerlo" (Ellis, 1997, p. 64) pueden repercutir en aspectos significativos de la enseñanza y aprendizaje de la L2. Entre las estrategias de feedback, una de las más estudiadas y que ha sido foco de interés para distintos teóricos es el feedback correctivo (CF) (Ferris, 2002; Joy, 2005; Sheen, 2011; Tatawy, 2002; entre otros). Este puede tener su origen en el contexto natural, donde es entregado por un hablante nativo a un hablante no nativo, quien pretende adquirir tal lengua como una L2; o en el contexto del aula, generalmente propiciado por el docente, quien cuenta con la facultad y el rol de optar por distintas estrategias en el marco de la enseñanza y aprendizaje de una L2. A nivel de la enseñanza de una L2, el CF es fundamental en el desarrollo de competencias gramaticales del idioma inglés. Por tanto, estas estrategias pueden, por una parte, potenciar los logros del estudiante y, por otra, orientarlo durante todo su aprendizaje.

La función del CF es proporcionar a los estudiantes información importante para que modifiquen los errores de sus enunciados. En suma, se entenderá por CF una indicación del profesor, ya sea explícita o implícita, que invita al aprendiz a atender un error gramatical específico, que demanda su corrección.

\section{Revisión de la literatura}

\section{Provisión de feedback a través de Google Docs}

La estructura de un ambiente colaborativo Google Docs ofrece grandes ventajas para la práctica de la 
producción escrita y para la provisión de feedback. El espacio virtual que ofrece Google Docs favorece el desarrollo de la competencia gramatical, la atención de los estudiantes, tanto en el feedback como en sus errores lingüísticos y el uso de sus habilidades lingüísticas para resolver problemas. Del mismo modo, el feedback otorgado al estudiante por el profesor o por un par, a través de un ambiente virtual, aumenta el número de revisiones y facilita el proceso (Ashwell, 2000; Braine, 2001; Liu y Sadler, 2003). Google Docs posee una aplicación que permite entregar comentarios en línea y facilita la labor del profesor durante la revisión del texto escrito. Lo anterior, dado que el feedback puede ser entregado a través de distintas instancias del proceso de escritura, de manera sincrónica y asincrónica.

El feedback y los cambios al texto escrito quedan registrados en Google Docs, por lo que los estudiantes pueden volver a este cuando lo estimen pertinente. Así, estas características de Google Docs facilitan aún más la corrección de errores y los avances en la escritura. Por consiguiente, se está frente a una modalidad de enseñanza que hace factible un aprendizaje mucho más dinámico para los estudiantes actuales, quienes tienen otras exigencias dado el contexto en el que se desenvuelven.

Algunos estudios relacionados con las percepciones de los estudiantes hacia el feedback señalan que, en general, existe una actitud positiva hacia el feedback en línea. Tuzi (2004) afirma que la provisión de feedback en línea estimularía la reflexión y la revisión frecuente de los textos escritos. Li (2000), en un estudio basado en el desarrollo de un texto en línea, llegó a la conclusión de que los estudiantes fueron más receptivos al feedback recibido de manera virtual que al convencional con lápiz y papel. También, Nezami (2012) determinó que el feedback en línea, principalmente del tipo recast y metalingüístico, era favorable para los estudiantes. Finalmente, Kelm (1996) y Warschauer (2010) plantean que los estudiantes demuestran una mejor disposición hacia el feedback entregado en un ambiente virtual. De esta manera, si bien estos resultados no son concluyentes, son diversas las ventajas que pueden ser encontradas al otorgar CF a estudiantes que conviven diariamente con la tecnología, a través de un espacio virtual.

\section{La corrección colaborativa de errores}

La revisión colaborativa de errores es concebida como una tarea que propicia la identificación de los errores en un texto escrito a través del diálogo y discusión entre los estudiantes (Memari, 2015). El diálogo facilita la disipación de dudas y resolución de problemas y, además, permite que los estudiantes utilicen la lengua para hablar de la propia lengua. Este es un aspecto que no es posible en una tarea individual, por tanto, adquiere gran importancia en la metodología colaborativa para la corrección de errores en una segunda lengua. Por otra parte, la corrección colaborativa de errores sitúa al estudiante dentro de un contexto en el que adquiere un rol más activo y autónomo, considerando que la revisión es una actividad comúnmente llevada a cabo por el docente.

Algunos estudios en este campo señalan que el trabajo colaborativo puede ser beneficioso para la detección de errores, puesto que, en un trabajo individual, algunos errores suelen pasar desapercibidos. En un estudio llevado a cabo por Liu y Kao (2007) se señala que los estudiantes reconocen la importancia de la colaboración en la detección de errores en sus textos escritos. Además, agregan que esta metodología promueve la formulación correcta de oraciones, la identificación de errores frecuentes, el aumento de su léxico y el reconocimiento de estructuras gramaticales. Los resultados de otro estudio realizado por Fernández y Blum (2013) indican que los estudiantes consideran que la metodología colaborativa, de una parte, promueve distintos puntos de vistas, la disipación de dudas y el desarrollo de la gramática y el vocabulario, y de otra, facilita la práctica del segundo idioma durante la discusión y resolución de un problema. Storch (2017), por su parte, declara que la metodología colaborativa facilita la adquisición de vocabulario y principalmente de la gramática, esto dado que los estudiantes se usan unos a otros como fuente de conocimiento. 
Un aspecto que es relevante destacar de la metodología colaborativa es su cualidad para activar lo metalingüístico durante el proceso de producción escrita. Al respecto, Camps, Guasch, Milian y Ribas (2000, p. 174) señalan que "las representaciones lingüísticas se construyen en el marco de la interacción social, ya que es a través de estas interacciones que se activa el aprendizaje lingüístico y la construcción del conocimiento". En este sentido, a través del trabajo en colaboración, el estudiante tomaría más conciencia sobre sus procesos mentales, lo que le ayudaría a conocer de mejor manera su propia forma de aprender. Algunos teóricos como Swain (1998), y Swain y Lapkin (2001) apoyan el uso de tareas colaborativas como una actividad que facilita la identificación de errores lingüísticos durante la fase dialógica. Esta instancia permite que los estudiantes hablen sobre la lengua y discutan sobre los errores lingüísticos. $\mathrm{Al}$ respecto, Swain (1995, p. 28) plantea que "las tareas de escritura colaborativa son efectivas para llamar la atención de los estudiantes hacia la forma, a pesar de que estas tareas están principalmente orientadas al significado". En concreto, la función social de la producción escrita y la activación de los procesos de reflexión y negociación del significado pueden facilitar el proceso de corrección de errores.

\section{Metodología}

\section{Preguntas de investigación}

¿Cuál es la percepción de estudiantes hacia la provisión de CF otorgado por el profesor a través de Google Docs?

¿Cuál es la percepción de estudiantes hacia la corrección colaborativa de errores?

\section{Tipo de estudio}

El presente es un estudio de caso de naturaleza interpretativa, puesto que se pretende indagar sobre un fenómeno a partir del estudio intensivo de varios casos.

\section{Muestra}

Se utilizó una muestra no probabilística intencionada de 44 estudiantes de segundo año de la carrera de Pedagogía Media en Inglés, de una universidad chilena. La experiencia se llevó a cabo en la asignatura de Competencia Comunicativa en Inglés II. La edad de los participantes fluctuaba entre los 19 y los 22 años, y su nivel de inglés corresponde a un B2 de acuerdo con el Marco Europeo de Referencias para las Lenguas.

Los criterios para la selección de la muestra fueron los siguientes:

1. Estudiantes de la carrera de Pedagogía en Inglés.

2. Nivel de inglés B2. Se consideró este nivel porque los participantes del estudio debían comprender la provisión de feedback indirecto en inglés.

\section{Instrumentos}

Para conocer las percepciones de los estudiantes acerca del efecto del CF otorgado por el profesor a través de un ambiente virtual y la corrección colaborativa de errores, se realizó una entrevista semiestructurada. Este instrumento consistió en diez preguntas divididas en dos dimensiones: percepción de los estudiantes hacia la provisión de feedback otorgado por el docente a través de Google Docs y percepción hacia la revisión colaborativa de errores. La entrevista fue aplicada a cada estudiante y tuvo una duración aproximada de 40 minutos.

\section{Técnica de análisis de datos}

Para el estudio de los datos, se empleó el análisis de contenido, que consiste en

[...] una técnica de recopilación de información que permite estudiar el contenido manifiesto de una comunicación, clasificando sus diferentes partes conforme a categorías establecidas por el investigador, con el fin de identificar de manera sistemática y objetiva dichas categorías dentro del mensaje. (Ander-Egg, 1995, p. 330) 
Inicialmente se establecieron categorías apriorísticas que emergieron del marco teórico. Posteriormente fueron ajustadas según la información que se obtuvo de las entrevistas.

Cada entrevista fue registrada en formato de audio $y$, posteriormente, se transcribieron los datos en documentos de Word. Luego, se utilizó el programa Nvivo 10 para organizar de los datos, seleccionar las unidades de análisis, codificar $y$, finalmente, seleccionar categorías y subcategorías.

\section{Procedimiento}

Con el fin de recoger las percepciones de los estudiantes, se llevó a cabo una experiencia en uno de los laboratorios de la universidad. Para esto, se contó con distintas fases las que son descritas en la siguiente tabla.

Tabla 1. Procedimiento de la experiencia

\begin{tabular}{|l|l|l|}
\hline \multicolumn{1}{|c|}{ Fase } & \multicolumn{1}{c}{ Descripción } \\
\hline 1 & Descripción de los objetivos de la tarea y de la metodología de trabajo. \\
\hline 2 & $\begin{array}{l}\text { Formación de los grupos por afinidad. Se determinó que cada grupo estuviera conformado por tres estu- } \\
\text { diantes. }\end{array}$ \\
\hline 3 & $\begin{array}{l}\text { Los estudiantes producen un ensayo argumentativo de manera colaborativa en Google Docs que consta de una } \\
\text { introducción, tres párrafos de desarrollo y una conclusión. }\end{array}$ \\
\hline & $\begin{array}{l}\text { Para la elaboración del escrito se asignan tareas grupales e individuales. En este contexto, los estudiantes de- } \\
\text { terminan primero en conjunto la tesis y las ideas que serán desarrolladas en cada párrafo. Luego, redactan en } \\
\text { conjunto la introducción y desarrollan cada párrafo individualmente, es decir, cada miembro del grupo queda } \\
\text { a cargo de un párrafo. Posteriormente, los estudiantes escriben la conclusión, al igual que la introducción la } \\
\text { desarrollan en grupo. Por último, revisan el texto en conjunto. }\end{array}$ \\
\hline 4 & $\begin{array}{l}\text { El profesor entrega cF indirecto en inglés a los escritos de los estudiantes. Por ejemplo, Technology plays an } \\
\text { important role in people's life. (El error es indicado sin entregar la respuesta correcta). }\end{array}$ \\
\hline 5 & Los estudiantes atienden al feedback entregado por el profesor y corrigen en conjunto el texto. \\
\hline 6 & Se aplica una entrevista semiestructurada.
\end{tabular}

Fuente: elaboración propia.

Como muestra la tabla 1, la experiencia se realizó en seis etapas. Estas comprendieron cuatro módulos de clase de 45 minutos cada uno. Para el control de la actividad, se decidió que esta se adelantara en el laboratorio de idiomas de la universidad.

\section{Resultados}

La tabla 2 contiene las categorías y subcategorías que surgieron de la revisión de las entrevistas, sobre el uso del entorno virtual Google Docs para la provisión de feedback y del trabajo en conjunto en la corrección de errores. 
Tabla 2. Categorías y subcategorías

Dimensión 1: provisión de feedback virtual por parte del profesor

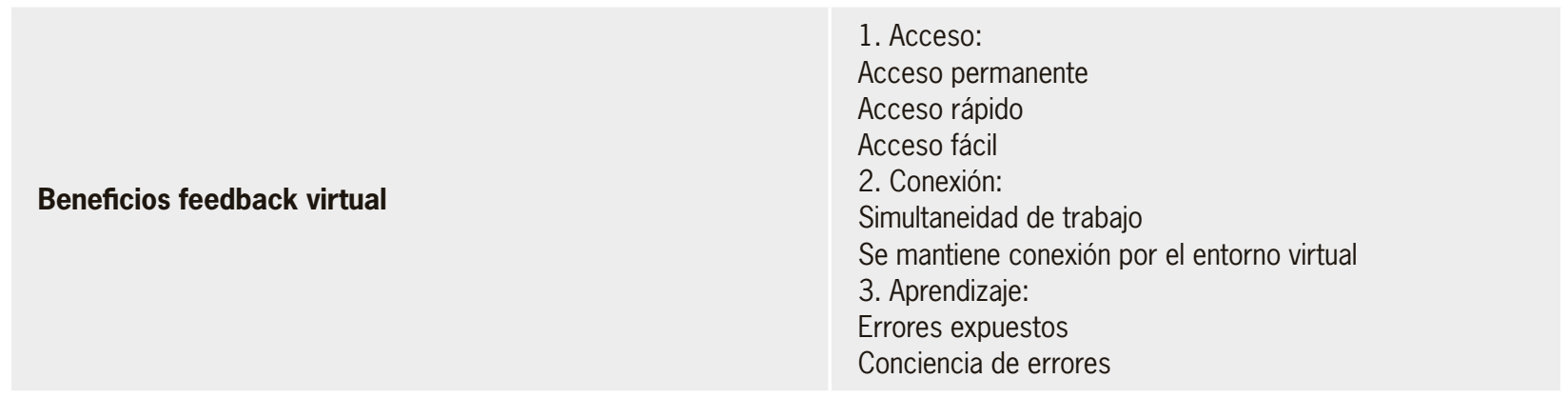

Dimensión 2: revisión colaborativa de errores

\begin{tabular}{|l|l|}
\hline \multicolumn{1}{|c|}{ Categoría } & \multicolumn{1}{c}{ Subcategoría } \\
\hline Beneficios del trabajo en conjunto & $\begin{array}{l}\text { Aprendizaje en conjunto } \\
\text { Discusión en grupo mejora calidad del trabajo } \\
\text { Conciencia de errores } \\
\text { Complementariedad } \\
\text { Facilita trabajo }\end{array}$ \\
\hline Desventajas trabajo en conjunto & $\begin{array}{l}\text { Aportes irregulares dentro de un grupo } \\
\text { Diferencias de conocimiento }\end{array}$ \\
\hline Preferencia por trabajo individual & $\begin{array}{l}\text { Preferencia por trabajo propio } \\
\text { Dificultad para trabajar en grupo por motivos personales }\end{array}$ \\
\hline
\end{tabular}

Fuente: elaboración propia.

\section{Discusión}

De las opiniones de los estudiantes surgen diversos análisis y reflexiones. En relación con los beneficios del feedback virtual los entrevistados enumeran varios, entre los que se destacan el acceso permanente, fácil y rápido, y la simultaneidad.

El entorno virtual representa una herramienta constante y presente, la que permite acceder al feedback con mucha facilidad y rapidez, sin la necesidad de material físico que se pierda. Esto se evidencia en las siguientes opiniones: "sí, porque era más fácil de revisar, está siempre ahí y podemos revisarlo entre varios a la vez y corregir en el momento"; "bueno, es más rápido, es mucho más rápido y más accesible y los errores se ven mejor, como usted los remarca"; "porque uno como que tiene acceso a la Web todo el tiempo, entonces si a uno le entregan un papel, por ejemplo, a mí se me pierde". El feedback en material impreso puede perderse a diferencia del que se encuentra en formato virtual que permanece en la
Web. Además, los estudiantes tienden a no corregir o reescribir un texto en la escritura tradicional.

Los estudiantes valoran de la herramienta virtual el hecho de que esté siempre disponible y que además les otorga la posibilidad de acceder a ella fácilmente y sin mayores obstáculos. Asimismo, la oportunidad que brinda un entorno virtual para trabajar de manera sincrónica es también altamente valorada por los estudiantes, ya que potencia el intercambio de información, optimiza el trabajo, permite una interacción y, por ende, un aprendizaje más fluido (Bartolomé, 2008; Cabero, 2007).

Esta aseveración se traduce en la siguiente opinión: "la posibilidad de realizar un trabajo al mismo tiempo con tu compañero o estar atento al feedback o a las correcciones del profesor sin salir de tu hogar, es una comodidad que entrega la conexión al entorno virtual". Esto permite una interacción con el docente más frecuente en comparación con el trabajo del aula y favorece la provisión de feedback. 
Del mismo modo, los estudiantes resaltan la accesibilidad como una característica relevante de Google Docs para la provisión de feedback, puesto que una herramienta como esta facilita una conexión más fluida con el docente, lo que favorece el aprendizaje. En la sala de clases es difícil que el docente pueda atender a cada necesidad individual. Esto se evidencia en las siguientes opiniones: "para la corrección de errores cumple bien su función, creo yo, además podíamos revisar en línea todos al mismo tiempo"; "el hecho de hacer trabajo en línea nos permitió trabajar uno por cada computador, aportando cada uno y al mismo tiempo". Mantener la conexión implica que el material puede revisarse en cualquier momento sin que cada estudiante deba tener su propio trabajo separado del otro. Dentro del mismo archivo y entre todos, es posible intercambiar ideas y correcciones, con todas las herramientas de búsqueda al alcance.

Por otra parte, algunos estudiantes demostraron una inclinación mayor por el feedback directo con el docente. Este hallazgo es compartido por otros estudios cuyos resultados que demuestran este hecho (Komura, 2009; Morgan y Toledo, 2006; Tuzi, 2004). Se podría erróneamente deducir que a raíz de la cercanía que tienen los estudiantes con las redes sociales, todos ellos se inclinarían más por un feedback virtual; sin embargo, tal vez por el hecho de que estén rodeados de tecnología, algunos valoran más el diálogo y el contacto más individualizado con el profesor. Es decir, un tipo de feedback en el que se produce una interacción activa entre estudiante y profesor (Dysthe, 2007).

Ahora bien, dada la realidad educativa, es necesario que los docentes promuevan en sus estudiantes la conciencia de que un feedback más personalizado puede alcanzarse únicamente si el contexto instruccional lo permite. La mayoría de los estudiantes no están acostumbrados a recibir un feedback virtual, debido, en gran medida, a que los docentes aún no están completamente familiarizados con estas herramientas. Por tanto, estos estudiantes necesitarán más tiempo para conocer las ventajas de Google Docs, lo que implica que los docentes los preparen y los familiaricen con nuevas formas de entregar feedback. Asimismo, algunos estudiantes valoran más el feedback individual, ya que necesitan recibir comentarios a sus propios errores.

En cuanto a los beneficios del trabajo en conjunto, y que apunta a la corrección colaborativa de errores de un ensayo argumentativo escrito de manera colaborativa, se desprende lo siguiente.

Los estudiantes entrevistados valoran que al realizar actividades grupales o en pareja se otorgue la oportunidad de aprender con sus compañeros, es decir, que entre ellos puedan hacer notar los errores del otro o mejorar ciertas habilidades y conocimientos que de otra manera no hubieran surgido. Esto se ve reflejado en las siguientes opiniones: "sí, porque, por ejemplo, yo a veces no entendía, entonces las compañeras me explicaban. Entonces tengo el apoyo de ellas, que me dicen aquí te equivocaste por esto y esto"; "si a uno le falta la idea o la palabra, la otra persona la tiene y es como la complementación de vocabulario y gramática igual”.

Lo anterior da cuenta de que los estudiantes se complementan cuando trabajan en grupo. El hecho de que dependan unos de otros para el logro de los objetivos es una forma de potenciar el aprendizaje de todos, puesto que se genera una dinámica en la que los estudiantes sienten que del esfuerzo y contribución individual depende el logro de las metas de los pares (Fernández y Blum, 2013). Ahora bien, puede ser difícil lograr este nivel de compromiso entre todos los integrantes de un grupo, pero es una forma de incentivar una mayor participación. Dicho grado de compromiso en la corrección de errores, después del feedback entregado por el profesor, es muy relevante, pues los alumnos, al ayudarse unos a otros para resolver sus dudas, prescinden del rol facilitador del docente y recurren a los pares para la resolución de problemas. En este mismo aspecto, los estudiantes señalan que la discusión mejora la calidad del trabajo. Esto se refleja en la siguiente opinión:

[...] cuando uno trabaja en grupo, a veces, cada uno está en su computador, podemos estar todos 
en línea trabajando en grupo y comentar los errores por chat y corregir en grupo, en eso es más eficiente. En la Web uno puede organizarse mejor con los grupos.

Por otra parte, la actividad colaborativa permitió que los estudiantes tuvieran mayor conciencia de los errores comunes que cometen regularmente. Esto los obliga a reunirse con los miembros del grupo para cumplir con los objetivos planteados y tomar decisiones. En una tarea individual, el estudiante no siente la presión del trabajo en equipo $\mathrm{y}$, por tanto, puede perfectamente no considerar el feedback entregado por el profesor, como ocurre generalmente en una tarea de escritura. Lo anterior se refleja en la siguiente opinión: "para mí fue mejor en grupo, porque, vuelvo a reiterar, el mismo hecho de que al recibir feedback individual hay errores que uno no ve y el otro compañero sí".

De esta manera, el trabajo en conjunto permite que cada miembro de un grupo sea un complemento importante para la redacción, la corrección y el aprendizaje en sí. Esto genera lo que se denomina interdependencia positiva entre los miembros del grupo, es decir, dependen unos de otros para el logro de los objetivos (Ferreiro, 2004).

Con respecto a las desventajas del trabajo en conjunto en la corrección de errores los estudiantes manifiestan que la principal desventaja se relaciona con los aportes irregulares que se dan dentro de un grupo, donde algunos aportan más que otros. En este contexto, un estudiante señala lo siguiente:

[...] encuentro que igual individual sigue siendo más completo, porque normalmente en un trabajo no siempre trabajan los dos las mismas partes o hay veces, si uno está apurado decía: "Yo hago esta parte, tú haces la otra", y al final como un Picasso medio extraño, entonces el feedback de repente no sé, yo revisaba y aparecían en un texto algo que no tenía coherencia supongamos, y lo había escrito mi otra compañera. Entonces yo lo leía y decía como: "Ya, ¿qué querría decir aquí?”. Entonces no, es más complicado en ese sentido.

En un caso habitual un estudiante puede trabajar muchísimo más que el otro (motivado por la nota final), mientras que su compañero puede realizar muy poco o nada y aun así llevarse el crédito del trabajo. A su vez, y como señalan London y Sessa (2006), un inconveniente que se puede presentar en el procesamiento del feedback es que los integrantes de un grupo reaccionen individualmente, o que tomen decisiones sin una discusión previa. Por otro lado, las combinaciones entre compañeros no siempre dan resultado, no siempre se entienden entre ellos o se complementan; así, la diferencia de conocimiento que existe entre ellos puede generar más roces que aprendizaje en conjunto. Lo anterior se refleja en la siguiente opinión:

[...] porque no todos los alumnos tienen un nivel de inglés tan alto como para saber qué tipo de error gramatical obtuvo en ese trabajo, entonces como hacer el feedback como una pregunta para que tú mismo te des cuenta de eso, como dije no todos los alumnos tienen el nivel de darse cuenta de eso.

Siguiendo con el análisis, algunos entrevistados manifestaron que por razones personales no les agrada trabajar en grupo o utilizar el entorno virtual, por lo que prefieren el trabajo individual. Lo anterior se refleja en las siguientes opiniones: "yo creo que ambas son necesarias. Pero a mí me gustaría más individual, porque, por ejemplo, yo estoy ansiosa de ver el último ensayo que hicimos a mano, porque siento que como que todo depende de mí"; "pero creo que el feedback tiene más valor para mí cuando es personalmente, porque se tiene una mayor conexión o una mayor preocupación del profesor". Al respecto, para algunos estudiantes, el aprendizaje es algo individual y por ello prefieren la revisión individual, aun considerando que el trabajo en conjunto no sea negativo.

Se aprecia que tales respuestas apuntan a una concepción más individual del aprendizaje, que nuevamente se contradice con las perspectivas actuales de entenderlo como un fenómeno más social. Comprender el aprendizaje como un fenómeno social, en el sentido que lo plantea Vygotsky (1999), puede no ser un objetivo fácil de comprender por los estudiantes. Sin embargo, es necesario abrirse a 
nuevos escenarios de aprendizaje y propiciar más instancias de colaboración en el aula para el desarrollo de la escritura y la corrección de errores. En este contexto, los estudiantes deben ser entrenados en el uso de metodologías vinculadas al trabajo colaborativo, las que van más allá de un simple trabajo en grupo. Asimismo, deben ser familiarizados con aprendizajes de tipo social y dialógico, con el fin de que asuman un rol más activo dentro de sus procesos de aprendizaje.

\section{Conclusiones}

La entrevista semiestructurada aplicada a los estudiantes permitió reflexionar sobre la importancia de la corrección de errores en inglés y sobre el impacto que puede tener el contexto en la provisión del feedback correctivo virtual. Al respecto, una de las conclusiones que se deriva de las opiniones de los estudiantes es la valoración positiva de estos hacia el feedback entregado a través de un medio virtual como Google Docs. En este contexto, las categorías y subcategorías que se derivaron del análisis ponen de manifiesto que Google Docs es una herramienta en línea que posee características que potencian el feedback. Tales beneficios no se encuentran en el feedback escrito entregado de manera tradicional. Por consiguiente, considerando la percepción positiva de la mayoría de los estudiantes entrevistados hacia un feedback entregado virtualmente, sería recomendable integrar esta metodología con mayor frecuencia dentro del proceso de enseñanza y aprendizaje para el desarrollo de la escritura colaborativa y para la entrega de feedback correctivo.

Por otra parte, ante la manifestación de algunos estudiantes por un feedback individual y más directo con el docente, se puede afirmar que una forma de satisfacer las necesidades de los estudiantes es combinar distintos tipos de feedback en distintos momentos, atendiendo además a que en la enseñanza intervienen varias combinaciones de opciones (Ellis, 2005). El uso de un feedback en línea, sumado a la provisión de un feedback individual, al menos una vez a cada estudiante, puede ser una buena combinación en el aula para atender a los errores que estos cometen y, así, satisfacer sus necesidades individuales.

En cuanto a la corrección colaborativa de errores, se concluye que los estudiantes mencionan más ventajas que desventajas; por ello, el docente debe recurrir a este tipo de metodologías con el fin de promover la autonomía, la discusión y facilitar la reflexión y disipación de dudas dentro de un grupo. Las nuevas herramientas tecnológicas, los enfoques basados en tarea y las metodologías colaborativas les asignan un rol más relevante a los estudiantes dentro del proceso de enseñanza y aprendizaje, y suscitan en ellos la interacción, la autonomía y la autorreflexión, lo que es, en general, percibido de manera positiva por ellos. Como afirma Shehadeh (2010), las actividades en parejas y en pequeños grupos entregan a los estudiantes mayor tiempo para hablar de la lengua, lo que influye en su autonomía y autoaprendizaje.

\section{Referencias}

Ander-Egg, E. (1995). Técnicas de investigación social. Editorial Lumen.

Ashwell, T. (2000). Patterns of teacher response to student writing in a multiple-draft composition classroom: Is content feedback followed by form feedback the best method? Journal of Second Language Writing, 9(3), 227-257.

Bartolomé, A. (2008). Entornos de aprendizaje mixto en educación superior. Revista Iberoamericana de Educación a Distancia, 11(1), 15-51.

Braine, G. (2001). A study of English as a foreign language (EFL) writers on a local-area network (LAN) and in traditional classes. Computers \& Composition, 18(2), 275-292.

Cabero, J. (2007). Las necesidades de las tic en el ámbito educativo: oportunidades, riesgos necesidades. Tecnología y Comunicación Educativas, 21(45), 4-19.

Camps, A., Guasch, O., Milian, M y Ribas, T. (2000). Metalinguistic activity: the link between writing and learning to write. En A. Camps y M. Milan (eds.), Metalinguistic activity in learning to write (pp. 103124). Amsterdam University Press. 
Dysthe, O. (2007). How a reform affects writing in higher education. Studies in Higher Education, 32(2), 237-252.

Ellis, R. (1997). Second language acquisition. Vol. 2. Oxford University Press.

Ellis, R. (2005). Principles of instructed language learning. System, 33(2), 209-224.

Fernández, A. y Blum, A. (2013). Collaborative writing in pairs and small groups. Learners' attitudes and perceptions. System, 41, 365-378.

Ferreiro, E. (2004). Alfabetización. Teoría y práctica. 6a. ed. Siglo XXI.

Ferris, D. (2002). Treatment of error in second language student writing. Ann Arbor: University of Michigan Press.

Ferris, D. y Roberts, B. (2001). Error feedback in L2 writing classes: How explicit does it need to be? Journal of Second Language Writing, 10(3), 161-184.

Joy, R. (2005). The effect of feedback and instruction on writing quality: legal writing and advanced L2 learners. [Tesis doctoral]. Washington University.

Kelm, O. (1996). The application of computer networking in foreign language education: Focusing on principles of second language acquisition. En Proceedings of the Hawaii Symposium Telecollaboration in Foreign Language Learning (pp. 19-28). University of Hawaii at Manoa, Second Language Teaching \& Curriculum Center.

Kessler, G. (2009). Student-initiated attention to form in Google Docs-based collaborative writing. Language Learning \& Technology, 13(1), 79-95.

Komura, K. (2009). Student response to error correction in ESL classrooms. [Tesis de maestría]. California State University, Sacramento.

Lightbown, P. y Spada, N. (2008). How languages are learned. Oxford University Press.

Li, Y. (2000). Linguistic characteristics of ESL writing in task-based e-mail activities. System, 28(2), 229-245.

Liu, C. y Kao, L. (2007). Do handheld devices facilitate face-to-face collaboration? Journal of Computer Assisted Learning, 23, 285-299.

Liu, J. y Sadler, R. (2003).The effect and affect of peer review in electronic versus traditional modes on $\mathrm{L} 2$ writing. Journal of English for Academic Purposes, 2(3), 193-227.
London, M. y Sessa, V. I. (2006). Group feedback for continuous learning. Human Resource Development Review, 5(3), 303-329.

Memari, A. (2015). Collaborative revision in L2 writing: Learner's reflection. ELT Journal, 70, 296-307.

Morgan, V. y Toledo, C. (2006). Online feedback and student perceptions. Journal of Interactive Online Learning, 5(3), 333-340.

Myles, J. (2002). Second language writing and research: the writing process and error analysis in student texts. TESL-EJ, 6(2), 1-20.

Nezami, S. H. (2012). A study of errors, corrective feedback and noticing in synchronous computer mediated communication. [Tesis de maestría]. http://urn.kb.se/ resolve?urn=urn:nbn:se:liu:diva-88411

Sheen, Y. (2011). Corrective feedback, individual differences and second language learning. Springer Publishing.

Shehadeh, A. (2010). One-to-one and group feedback in the L2 writing classroom: benefits and classroom implementation. En D. Anderson y C. Coombe (eds.), Cultivating real writers (pp. 109-120). Higher Colleges of Technology, United Arab Emirates.

Storch, N. (2017). Implementing and assessing collaborative writing activities in EAP classes. En J. Bitchener, N. Storch y R. Wette (eds.), Teaching writing for academic purposes to multilingual students. Instructional approaches (pp. 130-144). Routledge.

Swain, M. (1995). Three functions of output in second language learning. En G. Cook y B. Seidlhofer (eds.), Principle and practice in applied linguistics: studies in honour of H. G. Widdowson (pp. 125-144). Oxford University Press.

Swain, M. (1998). Focus on form through conscious reflection. En C. Doughty y J. Williams (eds.), Focus on form in classroom SLA (pp. 64-81). Cambridge University Press.

Swain, M. y Lapkin, S. (2001). Focus on form through collaborative dialogue: exploring task effects. En M. Bygate, P. Skehan y M. Swain (eds.), Researching pedagogic tasks: Second language learning, teaching and testing (pp. 99-118). Routledge.

Tatawy, M. (2002). Corrective feedback in second language acquisition, Columbia University Working Papers in TESOL \& Applied Linguistics, 2(2), 1-19. 
Universidad Pedagógica Nacional

Facultad de Humanidades

Tuzi, F. (2004). The impact of e-feedback on the revisions of L2 writers in an academic writing course. Computers and Composition, 21(2), 217-235.

Vygotsky, L. S. (1999). Tool and sign in the development of the child. En The collected works of L. S. Vygotsky.
Vol. 6. Scientific Legacy. Kluwer Academic/Plenum Publishers.

Warschauer, M. (2010). Invited commentary: new tools for teaching writing. Language Learning \& Technology, 14(1), 3-8. 